\title{
Modeling, Simulation and Control of a Loading Bridge
}

\author{
Marko Hančič, Gorazd Karer* , Igor Škrjanc
}

Faculty of Electrical Engineering, University of Ljubljana, Tržaška cesta 25, SI-1000 Ljubljana, Slovenia; *gorazd.karer@fe.uni-lj.si

Simulation Notes Europe SNE 25(3-4), 165 - 170

DOI: $10.11128 /$ sne.25.tn. 10309

Received: November 17, 2015;

Accepted: November 30, 2015

Abstract. This paper is focused on modeling, simulation and validation of the mathematical model of a loading bridge by comparison to the real system. A Lagrangian modeling method is presented to define a mathematical model of the loading-bridge dynamical system. Using the Matlab-Simulink simulation environment we have estimated the parameters and validated the model with regard to the experiments on the real device. The discrepancy between the simulated and the measured responses was adequate. Hence, we can conclude that the developed nonlinear model provides a satisfactory description of real system behaviour. Using the model, we designed a fuzzy controller for controlling load position and load height. The obtained controller has also been tuned and tested on the real device.

\section{Introduction}

In everyday engineering practice we are often faced with the problem of process-control design. When dealing with processes expressing relatively complex dynamic properties that are difficult to control or even dangerous when not taken care of properly, it is generally sensible to first describe the process as a mathematical model so as to be able to carry out the possibly problematic experiments and conduct the first stages of the control-design in a safe simulation environment. In this regard, we deal with a loading-bridge dynamical system. In order to be able to design a suitable controller and, in addition, to test the dynamical properties of the loading bridge, we have described the dynamics of the real device with a mathematical model, developed a simulator of the system and designed a fuzzy controller for the device.

\section{Description of the Loading Bridge}

The loading bridge Amira PS600 (see Figure 1) consists of an aluminium frame covered with sheets of plexiglass. Inside the frame, there is a guiding bar, along which the cart is driven by means of a DC-motor, a clutch, a tooth wheel and a tooth belt. The length of the bar is approximately $1.5 \mathrm{~m}$. Two proximity switches are mounted close to both ends of the guiding bar. They are used for limiting the position of the cart. The position of the cart is measured by an incremental encoder.

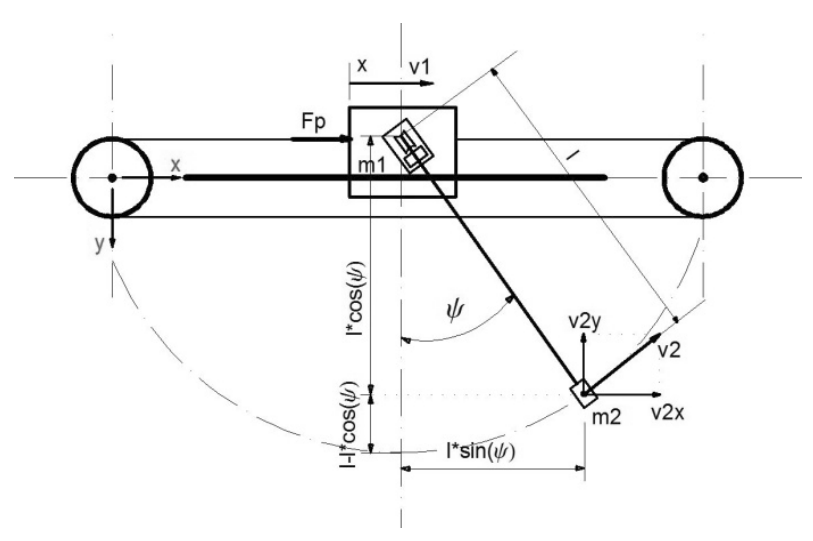

Figure 1: A scheme of the loading bridge with annotated quantities: $F_{p}$ - driving force on the cart, $\psi$ swing angle of the load, $I$ - rope length, $x$ - cart position, $m_{1}$ - cart mass, $m_{2}$ - load mass, $v_{1}$ - cart velocity, $v_{2}$ - load velocity, $v_{2 x}$ - horizontal component of load velocity, $v_{2 y}$ - vertical component of load velocity.

The cart carries a winch that is used to change the length of the rope. The rope runs around two deflection rollers and carries the load, which is suspended below the guiding bar. The length of the rope is measured by an incremental encoder. 
The loading bridge allows the load to swing in parallel to the guiding bar when the cart is moved to the left or to the right. The swing angle is measured by an incremental encoder [3]. Table 1 shows the measured parameters of the loading bridge.

\begin{tabular}{cc}
\hline Parameter & Value \\
\hline $\mathbf{r}$ & $0.3183 \mathrm{~m}$ \\
\hline $\mathbf{m}_{\mathbf{t}}$ & $0.2 \mathrm{~kg}$ \\
\hline $\mathbf{m}_{\mathbf{1}}$ & $5.5 \mathrm{~kg}$ \\
\hline $\mathbf{m}_{\mathbf{2}}$ & $0.143 \mathrm{~kg}$ \\
\hline
\end{tabular}

Table 1: The measured parameters of the loading bridge. Here, $r$ is the radius of the winch, $m t$ is the mass of the winch, $\mathrm{ml}$ denotes the mass of the cart and $\mathrm{m} 2$ the mass of the load.

\section{Derivation of the}

\section{Mathematical Model}

The mathematical model of the loading bridge was derived using the Lagrangian method [1,2]. A general Lagrangian partial differential equation is given in eq. (1).

$$
\frac{d}{d t}\left(\frac{\partial L}{\partial \dot{q}_{s}}\right)-\frac{\partial L}{\partial q_{s}}+\frac{\partial P}{\partial \dot{q}_{s}}=F_{s}
$$

Here, $q_{s}$ is a generalized coordinate describing the model of the system. $P$ represents the power-dissipation function - see eq. (2) - where $R$ is the damping factor.

$$
P=\frac{1}{2} R \dot{q}_{s}^{2}
$$

$L$ stands for the Lagrangian in eq. (3).

$$
L=T_{k}-V_{p}
$$

Here, $T_{k}$ is the kinetic energy of the system and $V_{p}$ represents the potential energy of the system. $F_{S}$ stands for the external excitation along the respective generalized coordinate. By considering the kinetic and potential energy of the system, the Lagrangian can be rewritten as in eq. (4).

$$
\begin{aligned}
L=\frac{1}{2} \dot{x}^{2}\left(m_{1}+m_{2}\right) & +\frac{1}{2} \dot{\psi}^{2} l^{2} m_{2}+\frac{1}{2} \dot{l}^{2} m_{2} \\
& +\dot{x} m_{2} \sin (\psi)+\dot{x} \dot{\psi} l m_{2} \cos (\psi) \\
& +\frac{1}{2} J\left(\frac{\dot{i}^{2}}{r^{2}}\right)+m_{2} g l \cos (\psi)
\end{aligned}
$$

Here, $J$ is the moment of inertia of the winch, $r$ is its radius and $g$ represents gravitational acceleration.

Based on eq. (1) a partial differential equation for each of the generalized coordinates can be established. In our case, there are 3 generalized coordinates, i.e.: cart position $x$ in eq. (5); swing angle $\psi$ in eq. (6); and rope length $l$ in eq. (7):

$$
\begin{aligned}
& \frac{\partial}{\partial t}\left(\frac{\partial L}{\partial \dot{\mathbf{x}}}\right)+\frac{\partial L}{\partial x}+\frac{\partial P}{\partial \dot{\mathbf{x}}}=F_{p} \\
& \frac{\partial}{\partial t}\left(\frac{\partial L}{\partial \dot{\psi}}\right)+\frac{\partial L}{\partial \psi}+\frac{\partial P}{\partial \dot{\psi}}=0 \\
& \frac{\partial}{\partial t}\left(\frac{\partial L}{\partial \dot{\mathrm{l}}}\right)+\frac{\partial L}{\partial \mathrm{l}}+\frac{\partial P}{\partial \dot{\mathrm{l}}}=T
\end{aligned}
$$

Upon inserting the Lagrangian and the powerdissipation function into eqs. (5), (6) and (7) and carrying out the derivations, we obtain the following $2^{\text {nd }}$ order differential equations for $x, \psi$ and $l$ in eqs. (8), (9) and (10), respectively.

$$
\begin{aligned}
& \ddot{x}=\left(m_{1}+m_{2}\right)^{-1}\left[F_{p}-R_{t r} \dot{x}-\ddot{l} m_{2} \sin (\psi)\right. \\
& -2 \dot{\psi} l \dot{m}_{2} \cos (\psi)-\ddot{\psi} l m_{2} \cos (\psi)(8) \\
& \left.+\dot{\psi}^{2} \operatorname{lm}_{2} \sin (\psi)\right] \\
& \ddot{\psi}=l^{-1}\left[-\ddot{x} \cos (\psi)-g \sin (\psi)-2 \dot{\psi} \dot{l}-R_{p s i} * \dot{\psi}\right] \\
& \ddot{l}=\left(m_{2}+\frac{J}{r^{2}}\right)^{-1}\left[T-R_{t r l} \dot{l}-\ddot{x} m_{2} \sin (\psi)\right. \\
& \left.+\dot{\psi}^{2} l m_{2}+m_{2} g \cos (\psi)\right]
\end{aligned}
$$

The aforementioned equations define the mathematical representation of the system.

\section{Simulation and Validation of the Mathematical Model}

The nonlinear mathematical model of the loading bridge was implemented in the Matlab/Simulink simulation environment. The simulation scheme of the nonlinear model is presented in Figure 2. 


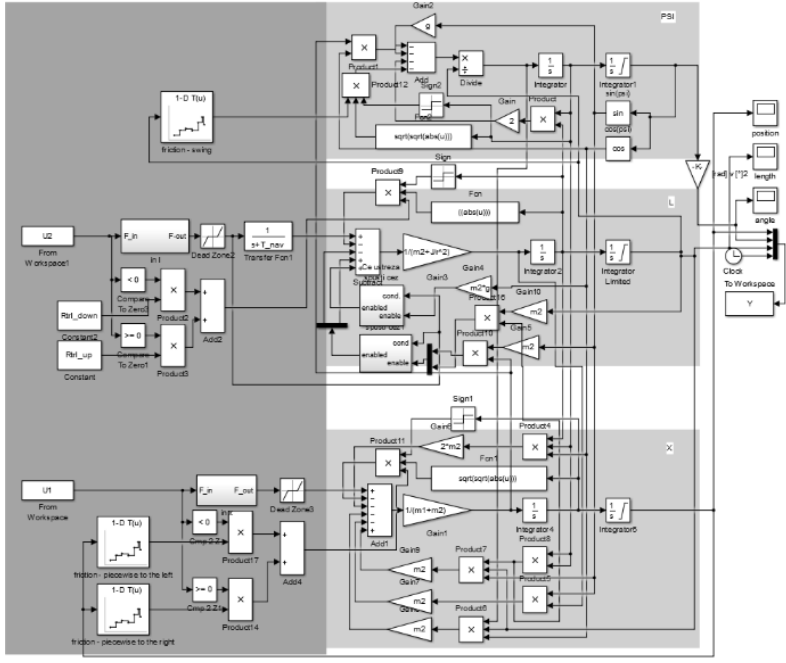

Figure 2: Simulation scheme of the nonlinear model of the loading bridge.

In order to validate the obtained nonlinear model, the responses of the model were compared to the real loading-bridge response-signal measurements using the scheme in Figure 3.

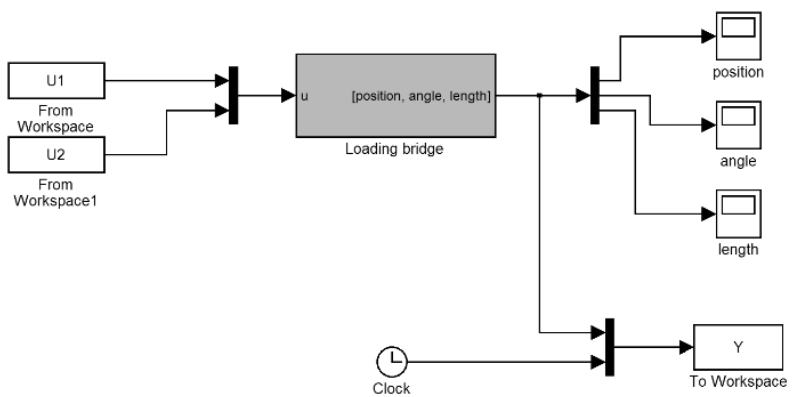

Figure 3: Measurement scheme for acquiring real loadingbridge response signals.

\subsection{Parameters of the nonlinear model}

The real loading bridge and the developed nonlinear model were fed a positive and negative step-signal for the cart-motor input and the appropriate response signals were recorded (see Figure 4 - left). Figure 4 depicts the comparison of signals of the nonlinear model and the measured signals on the real plant. We can see that the cart does not return to the initial position (see Figure 4 - right).
The dynamical model had to be adjusted in order to close the discrepancy gap between the model and the real device response signals. The friction parameters are not constant along the guiding bar. In addition, they depend on the direction the cart is moving as well. Therefore, a series of measurements was conducted, taking into account the relevant partitions of the guiding bar. A testing input signal was used that moved the cart to the left and to the right. Descending and ascending ramps were used for the measurements, respectively. The respective ramps were time-limited and amplitudelimited.

The distance travelled was used to estimate the friction parameters appurtenant to the relevant partition of the guiding bar. In such a manner, the developed model includes several lookup tables that describe the nonlinear dynamics within a particular partition. The main cause for nonlinearity is non-constant friction along the guiding bar and a dead-zone of the input signal that provides the voltage to the motor.

In order to estimate the damping properties of the pendulum, which is made up of the load and the loadcarrying rope, several responses to the initial nonequilibrium condition were measured. The measurements were initially carried out without moving the cart along the guiding bar. The responses were measured with various lengths of the rope $l$. The damping coefficient was adjusted until the response of the nonlinear model matched the response of the real device.
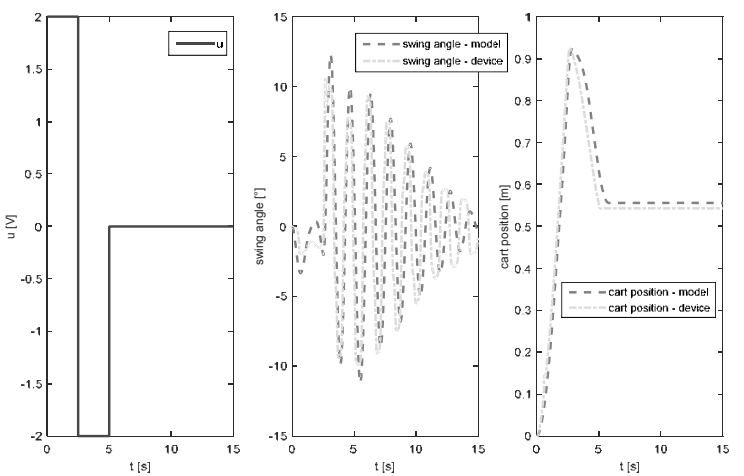

Figure 4: Validation input signal (positive and negative steps: $2 \mathrm{~V}$ and $-2 \mathrm{~V}$, respectively) for the cart and response comparison between the nonlinear model (blue line) and the real device (red line). The rope length was constant $(I=0.7 \mathrm{~m})$. 
The responses regarding the change of the rope length as a consequence of the input signal of the winch were also adjusted. In order to match the response of the model to the response of the real device, a dead-zone within the input-voltage has to be considered. Furthermore, the winch submodel includes a $1^{\text {st }}$ order delay. The measurements suggest that the discrepancy between both response signals is acceptable.

\section{Tracking Control of the Load Along the Reference Trajectory}

Tracking control of the load along the reference trajectory was designed by implementing two fuzzy controllers. One was used to control the position of the cart and the other one controlled the length of the rope that carries the load. The reference trajectory is automatically generated from the initial and the final position within Matlab.

The fuzzy controller for the cart position has 3 input variables and 1 output variable, whereas the fuzzy controller for the rope length has 2 input variables and 1 output variable. Both are Mamdani type controllers with centroid defuzzyfication. Table 2 shows the input and output variables and the respective ranges for both fuzzy controllers.

\begin{tabular}{ccccc}
\hline \multirow{2}{*}{ Controller } & Variable & $\begin{array}{c}\text { Lower } \\
\text { Limit }\end{array}$ & $\begin{array}{c}\text { Upper } \\
\text { Limit }\end{array}$ & Unit \\
\hline \multirow{4}{*}{$\begin{array}{c}\text { Cart } \\
\text { position - } x\end{array}$} & Error $\mathrm{x}_{\mathrm{r}}-\mathrm{x}$ & -3 & 3 & {$[\mathrm{~m}]$} \\
\cline { 2 - 5 } & Velocity $\dot{x}$ & $-0,7$ & 0,7 & {$[\mathrm{~m} / \mathrm{s}]$} \\
\cline { 2 - 5 } & Swing angle $\Psi$ & -45 & 45 & {$\left[{ }^{\circ}\right]$} \\
\cline { 2 - 5 } & Motor voltage & -3 & 3 & {$[\mathrm{~V}]$} \\
\hline \multirow{3}{*}{ Rope length - I } & Error $\mathrm{I}_{\mathrm{r}}-\mathrm{I}$ & -3 & 3 & {$[\mathrm{~m}]$} \\
\cline { 2 - 5 } & Velocity $i$ & $-0,7$ & 0,7 & {$[\mathrm{~m} / \mathrm{s}]$} \\
\cline { 2 - 5 } & Motor voltage & -10 & 10 & {$[\mathrm{~V}]$} \\
\hline
\end{tabular}

Table 2: Input and output variables and their respective ranges for both fuzzy controllers.

In both cases, the errors are normalized to the interval $[-1,1]$, so that the controller is comparably responsive within the operating range.
Figure 5 depicts the membership function of the fuzzy controller for cart position, whereas Figure 6 shows the membership function of the fuzzy controller for rope length. The membership functions regarding the error in both cases are condensed around 0 , which ensures high accuracy of control.

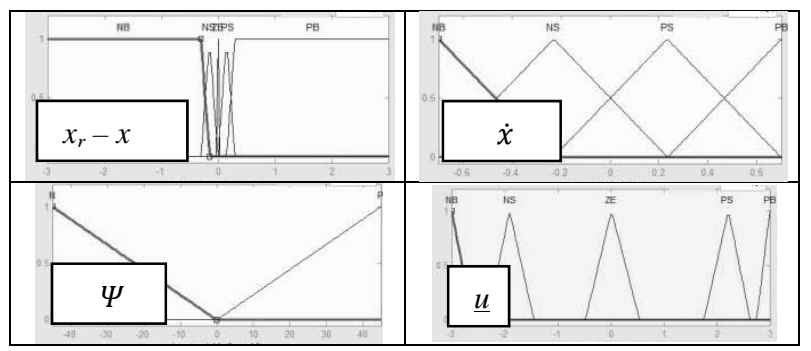

Figure 5: Membership functions of the input and output variables of the fuzzy controller for cart position. Trapezoidal, triangular and Gaussian membership functions are used.

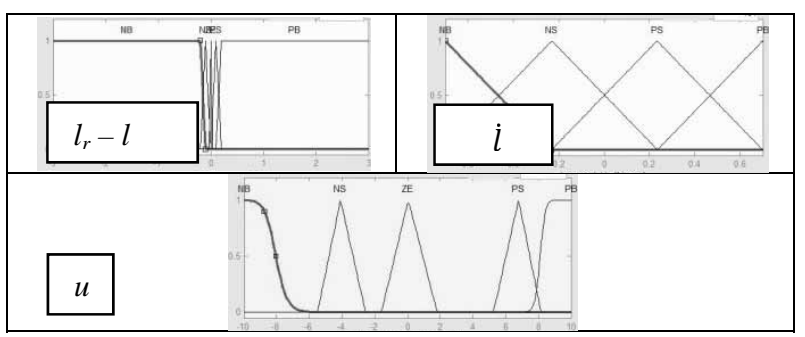

Figure 6: Membership functions of the input and output variables of the fuzzy controller for rope length. Trapezoidal, triangular and Gaussian membership functions are used.

The inputs and outputs of each fuzzy controller are connected by an inference system, which are defined by appropriate rules, as defined below:

1. If (Error is $N B$ ) then (Voltage is $N B$ ).

2. If (Error is $P B$ ) then (Voltage is $P B$ ).

3. If (Error is NS) then (Voltage is NS).

4. If (Error is PS) then (Voltage is PS).

5. If (Error is NS) and (Velocity is PB) then (Voltage is NS).

6. If (Error is PS) and (Velocity is NB) then (Voltage is $P S)$.

7. If (Error is ZE) then (Voltage is ZE).

8. If (Angle is $N$ ) then (Voltage is ZE).

9. If (Angle is $P$ ) then (Voltage is $Z E$ ). 
Both controllers employ similar rules, except for rules 8 and 9, which are not considered in the controller for rope length. On one hand, rules from 1 to 7 tend to minimize the error by appropriately adjusting the motor voltage. On the other hand, rules 8 and 9 are used to slow down the cart to prevent the swing angle of the load exceeding safe boundaries.

Figure 7 shows the control scheme where both fuzzy controllers are implemented. The reference trajectory was generated from the initial position of the load $T_{z}=[0,0.185]$ along the first reference goal-point $T_{1}=[1.2,0.6]$ and ending up in the final reference goalpoint $T_{2}=[0.5,0.3]$.

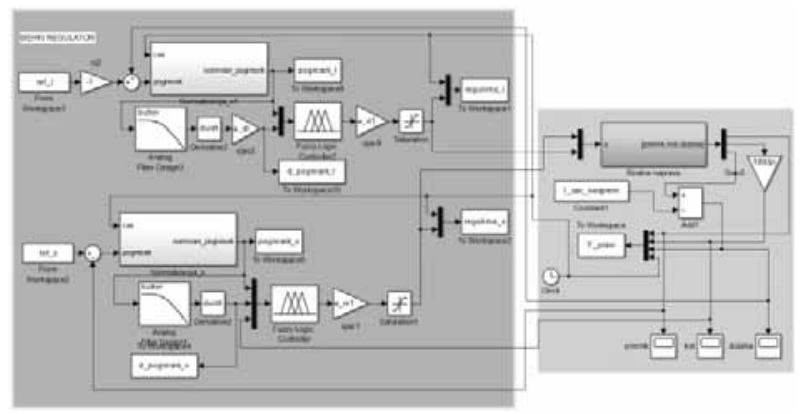

Figure 7: Control scheme with two fuzzy controllers. The same controllers are implemented in the simulation experiment using the nonlinear model and in the experiment using the real device.

The experiments are carried out by first moving the load from the initial position $T_{z}$ to the first reference goalpoint $T_{l}$ and then moving the load to the final reference goal-point $T_{2}$. Figure 8 depicts the comparison between the responses of the model (red line) and of the real device (green line), with regard to the reference trajectory (blue line). The measurements suggest that the discrepancy between the responses is quite small.
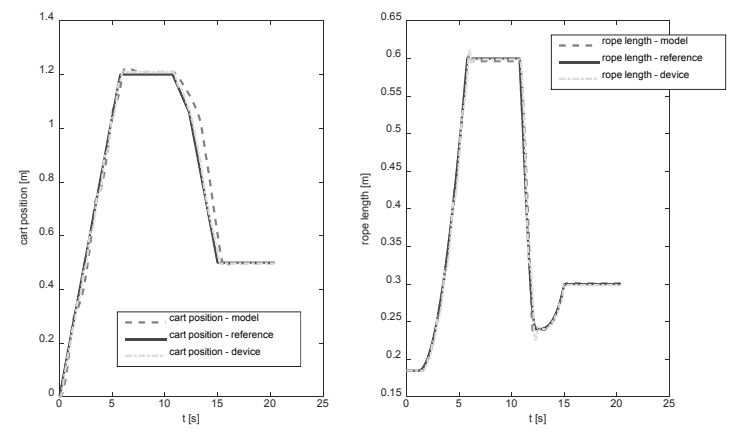

Figure 8: Comparison between the responses of the model (red line) and of the real device (green line), with regard to the reference trajectory (blue line).
Figure 9 shows the control signals for the cart position (left) and for the rope length (right). Red line denotes the signals of the nonlinear model, whereas green line is for the signals concerning the real device.
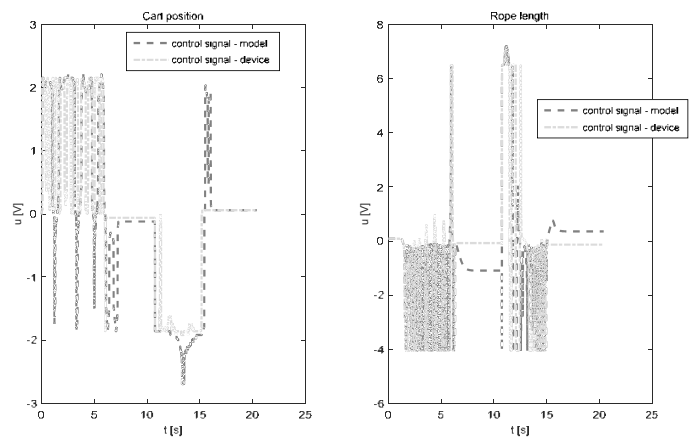

Figure 9: Control signals for cart position (left) and for rope length (right). Red line - nonlinear model; green line - real device.

The behaviour of the simulated model and of the real device are rather similar, although the actuators of the real device are slightly more loaded. Reference tracking of the cart position is favourable both in simulation and on the real device, despite noticing slight overshoots and a negligible steady-state error. Reference tracking o the rope length is also satisfactory in both cases, in spite of minor overshoots. Figure 10 depicts the trajectory of the load from $T_{z}=[0,0.185]$ along $T_{1}=[1.2,0.6]$ to $T_{2}=[0.5,0.3]$.

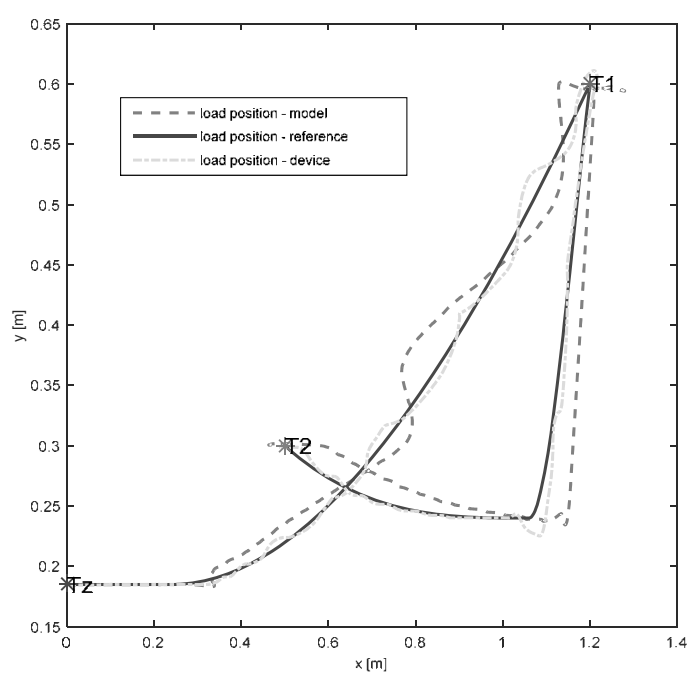

Figure 10: Trajectory of the load: reference (blue line), simulated model response (red line), real device response (green line). $T_{z}=[0,0.185]$ - initial position; $T_{1}=[1.2,0.6]$ - first reference goal-point; $T_{2}=[0.5,0.3]-$ final reference goal-point. 


\section{Conclusion}

The paper illustrates the development of the mathematical model of the loading bridge using the Lagrangian modeling method. The first iteration of the developed model does not fully encompass the dynamics of the real device due to nonmodeled phenomena and repeatability issues. Therefore, the model has been enhanced in order to close the gap between its simulated responses and the responses of the real device.

Two fuzzy controllers - i.e., one for the cart position and ono for the rope length - have been designed and tested. The structure of both controllers is similar, although two additional rules are implemented in the controller for cart position so as to limit the swing angle of the suspended load. Simulation experiments and experiments with the real device lead to conclusion that good control performance can be achieved using the designed fuzzy controllers when tracking the reference trajectory of the load position. Furthermore, the developed model provides the basis for a safe and convenient environment for testing various control strategies. Successful experiments assure that the developed controles can be adequately implemented on the real loading bridge.

\section{References}

[1] Atanasijevič Kunc M. Metode modeliranja-Zapiski predavanj, Faculty of Electrical Engineering, University of Ljubljana, 2013.

[2] Atanasijevič Kunc M. Modeliranje procesov: zbirka primerov z ilustracijami v okolju Matlab-Simulink, Faculty of Electrical Engineering, University of Ljubljana, 2008.

[3] Amira PS600 V2.0; Laboratory Experiment Loading Bridge. Amira GmbH, Duisburg, 2000.

[4] Karba R. Modeliranje procesov, Založba FE in FRI, University of Ljubljana, 1999.

[5] Zupančič B, Karba R., Matko D., Škrjanc I. Simulacija dinamičnih sistemov, Ljubljana, Založba FE in FRI, 2010. 